\title{
Aconitine mediates connexin43 and PKCa phosphorylation status in cultured ventricular myocytes of neonatal rats
}

\author{
Yan Liu', Man Liang ${ }^{1}$, Shi-wei Zhang ${ }^{2}$, Lan Zhou ${ }^{3}$, Chuan-hong Zhu ${ }^{4}$ and Liang Liu ${ }^{5 *}$ \\ ${ }^{1}$ Department of Forensic Medicine, Tongji Medical College of Huazhong University of Science and Technology, \\ Wuhan 430030, China. \\ ${ }^{2}$ Zhongshan Hospital of Hubei Province, Wuhan 430033, China. \\ ${ }^{3}$ Institute of Forensic Science, Bureau of Public Security for Jiangsu Province, Nanjing 210024, China. \\ ${ }^{4}$ Wuhan Municipla Public Security Bureau, Wuhan 430029, China. \\ ${ }^{5}$ Key Laboratory of Evidence Science (China University of Political Science and Law), Ministry of Education, \\ Beijing 100040, China.
}

Accepted 5 April, 2011

\begin{abstract}
Aconitine, a strong poisonous type of alkaloid, has a pharmaceutical effect in stimulating the membranes of cardiomyocyte. However, other effects of aconitine on the Connexin43 (Cx43) and PKCa expression on cardiomyocyte are unknown. In this study, we investigated whether aconitine also mediates the phosphorylation status of $\mathrm{Cx43}$ and PKC $\alpha$ in cultured ventricular myocytes of neonatal rats. The band intensity of phosphorylated $\mathrm{Cx} 43$ and nonphosphorylated Cx43 in cultured and aconitine-treated cardiomyocytes were determined by Western blot analysis. The changes in phosphorylation status occurring in PKC $\alpha$ in cultures were revealed by quantitative immunofluorescent microscopy. A decreased band intensity $(0.37 \pm 0.04)$ of phosphorylated Cx43 (P-Cx43) and a concomitant increased band intensity (3.56 \pm 0.65 ) of nonphosphorylated Cx43 (NP-Cx43) were found, compared to the controls (1.00 for P-Cx43 and NP-Cx43). It also revealed that, after aconitine treatment, the amount of phosphorylated PKCa (P-PKCa) decreased significantly. Similar changes were revealed in phosphorylation status occurring in PKCa in the cultures under the same treatment conditions. These observations suggest that aconitine not only induces dephosphorylation of $\mathrm{Cx} 43$, but also alters expression of P-PKC $\alpha$ in cultured cardiomyocytes.
\end{abstract}

Key words: Aconitine, cardiomyocyte, connexin 43 (Cx43), protein kinase C- $\alpha$ (PKCa), protein phosphorylation.

\section{INTRODUCTION}

Aconitium plants are typical series of officinal toxic traditional Chinese drug. Aconitine, the main effective constituent of Aconitium plants, is of effects such as cardiotonic, analgesia, anti-inflammatory, anti-tumor and so on, which contribute to widespread use in clinical (Baselt, 2004). Related reports of poisoning, even death caused by improper processing, misuse or accidental

\footnotetext{
${ }^{*}$ Corresponding author. E-mail: casper603@gmail.com.
}

ingestion are matters of common occurrences; however, cases of suicide and homicide meet the same condition according to forensic identification (Elliott, 2002; Pullela et al., 2008; Chan., 2009). Aconitine suppresses the deactivation of voltage-gated sodium channels, which prevents complete repolarization of the excitable membrane of cardiac tissues (Liu et al., 2008; Wang et al., 2003; Moric, et al., 2003; Li et al., 2007), all which caused aconitine-induced arrhythmias. Such previous conclusions based on aconitine-induced abnormal electrical activity in vivo and in vitro by clamp method (Amran et al., 
2004); nonetheless, the involvement of the isolated single cell plays an insignificant role in the aconitine-induced arrhythmias due to syncytial structure and function of cardiomyocytes.

Gap junctions, laid between membranes of adjacent myocardium, were composed of connexin (Cx) protein subunits. The formed conduits between adjacent cells coordinate intercellular electrochemical communication and ensure the transfer of large amounts of molecules to selectively pass from one cell to another (Kumar et al., 1996; Sohl and Sohl, 2004; Giepmans, 2004; Sáez et al., 2005). To date, serine residues of Cx43 (Ser368) is the most important phosphorylated modification site in the cardiovascular system and it is closely related to deletion of myocardium electrical coupling and poor contraction induced by ischemia and hypoxia (Dhein et al., 2002; Schulz and Heusch, 2004; Lin et al., 2006).

Since the protein kinase C- $\alpha$ (PKCa) was a type of $\mathrm{Ca}^{2+}$ and phospholipids-dependent endoenzyme of signal pathway and it was reported to have a signal transduction part in regulating the phosphorylation of $\mathrm{Cx} 43$, which eventually affect the function of cardiac gap junctions (Dhein et al., 2001; Weng et al., 2002).

Cardiac gap junctions are known to be particular conduction of the electrical impulse among cardiomyocytes, and $\mathrm{Cx} 43$ is the most important gap junction protein in the cardiac ventricles (Salameh, 2006; Xia et al., 2009). And $\mathrm{C} \times 43$ is a phosphorprotein, which highly regulated by different protein kinases at different serine or tyrosine residues in its carboxyl terminal domain. There are reports that electrical uncoupling induced by myocardial ischemia is associated with Cx43 dephosphorylation of Ser368 (Beardslee et al., 2000). These pieces of evidence indicate that aconitine-induced electrical uncoupling and arrhythmia among cardiomyocytes could be related, at least in part, to Cx43 dephosphorylation. It is reported that the antiarrhythmic peptide (AAP) can improve cellular coupling and increase cardiac gap junction conductance, which could release arrhythmia induced by aconitine and so on (Axelsen et al., 2006; Stahlhut et al., 2006). In this work, we investigated the effects of aconitine on $\mathrm{Cx} 43$ phosphorylation status and PKCa in cultured ventricular myocytes of neonatal rats. In this respect, we also evaluated the mechanisms underlying these aconitine-induced effects.

\section{MATERIALS AND METHODS}

\section{Preparations of the drugs and experiment conditions}

Aconitine was subscribed from the National Institute for the Control of Pharmaceutical and Biological Products (Beijing, China, 110720). 5, 6, 7, 13-Tetrahydro-13-methyl-5-oxo-12H-indolo [2, 3-a] pyrrolo [3, 4-c] carbazole-12-propanenitrile (Gö6976) was purchased from Sigma (USA). Antiarrhythmic peptide (AAP) was purchased from United States Biological (Swampscott, MA, USA, A2298-11C). All of the drug stock solutions $(100 \mathrm{x})$ were prepared according to the manufacturers' instructions and diluted in serum-free DMEM to the desired concentrations immediately before use. The final concentration of DMSO was less than $0.05 \%$, which had no significant effect on the experiments.

\section{Cell isolation, culture and protein extraction}

The ventricles of 1-day-old Sprague-Dawley rats were prepared for primary cultures. After remove, hearts were placed in ice-cold Hanks' balanced salt solution (HBSS, without calcium chloride or magnesium sulfate, Sigma, St. Louis, MO, USA) and washed twice. The isolated ventricles were minced and digested in HBSS containing $0.8 \mathrm{mg} / \mathrm{ml}$ trypsin (Sigma), $1 \mathrm{mg} / \mathrm{ml}$ collagenase type II (Invitrogen-GIBCO, Carlsbad, CA, USA) and $0.5 \mathrm{mg} / \mathrm{ml}$ bovine serum albumin (Sigma) by stirring at $37^{\circ} \mathrm{C}$ for $10 \mathrm{~min}$; the first supernatant containing cell debris and blood cells was discarded. Then the digestion was repeated three times; the cell suspension was collected. Cell suspensions from the three subsequent digestions were centrifuged at $4^{\circ} \mathrm{C}$ and $1000 \mathrm{rpm}$ for $10 \mathrm{~min}$, then the resultant pellet was resuspended in $3 \mathrm{ml}$ of Dulbecco's modified Eagle's medium (DMEM, with $4500 \mathrm{mg} / \mathrm{L}$ glucose, L-glutamine, and $25 \mathrm{mM}$ HEPES, Sigma), containing 15\% fetal bovine serum (FBS, GIBCO), and the process was repeated once again. Cells were pooled and maintained in incubator, containing complete DMEM supplemented with $100 \mathrm{U} / \mathrm{ml}$ penicillin, $100 \mu \mathrm{g} / \mathrm{ml}$ streptomycin, 2 $\mathrm{mM}$ glutamine (GIBCO) and $15 \%$ FBS, for $1 \mathrm{~h}$ at $37^{\circ} \mathrm{C}$. The incubation induced selective attachment of nonmyocytes. Then the unattached cells were seeded at a density of $1 \times 10^{4} \mathrm{cell} / \mathrm{cm}^{2}$ on 12-well culture plates (Corning Transwell ${ }^{\circledR}$ insert, Acton, MA, USA), which was pre-coated with collagen type-I (Sigma) and prewarmed at $37^{\circ} \mathrm{C}$, in $5 \% \mathrm{CO}_{2} .0 .1 \mathrm{mM} \mathrm{5-BrdU} \mathrm{(Sigma-Aldrich)} \mathrm{was} \mathrm{used} \mathrm{to}$ inhibit the growth of fibroblast in the medium. After $24 \mathrm{~h}$ incubation, a confluent monolayer of cardiomyocytes had developed, slowly and synchronously beating. Cultured cells were rinsed and replenished with fresh DMEM containing 10\% FBS at the moment, then the medium was replenished every $48 \mathrm{~h}$; and the procedures were repeated three times in 6 days.

After equilibrating the cells under serum-free conditions for 16 to $18 \mathrm{~h}$, subsequent experiments were performed on. During preliminary experiments, we had identified the cultures using anti-cTnl antibodies (Abcam, Cambridge, UK; ab19615) and estimated that most of the cultured cells were cardiomyocytes. Cultures were treated with aconitine $(1 \mu \mathrm{M}$, for $1 \mathrm{~h})$, AAP $(0.1 \mu \mathrm{g} / \mathrm{ml}$, for $4 \mathrm{~h})$ and Gö6976 $(0.1 \mu \mathrm{g} / \mathrm{ml}$, for $4 \mathrm{~h})$, respectively; or pretreated with AAP for $4 \mathrm{~h}$, followed by $1 \mathrm{~h}$ of aconitine incubation; or co-incubated with AAP and Gö6976 under the same concentration conditions. All the incubation groups were used in both quantitative Western blot analyses of $\mathrm{Cx} 43$ and $\mathrm{PKCa}$ and quantitative immunofluorescent microscopy analysis.

The treated cultures were rinsed with ice-cold HBSS, $1 \mathrm{~min} x$ twice, and harvested by scraping. A membrane protein extracting kit (BioVision, Mountain View, CA, USA, K268-50) was used according to the recommended protocol. The pellets of cellular membrane protein were dissolved in PBS and diluted to a concentration of 10 $\mu \mathrm{g} / \mu \mathrm{l}$, using BCA protein assay kit (Pierce, Rockford, IL, USA, 23227). The prepared samples were stored at $-80^{\circ} \mathrm{C}$ for further use.

\section{Quantitative western blotting}

Aliquots, containing $30 \mu \mathrm{g}$ sample protein, were mixed into a NuPAGE LDS sample buffer (Invitrogen, USA, WG1602), containing $1 \mu \mathrm{l}$ reducing agent, and denatured at $70^{\circ} \mathrm{C}$ for $10 \mathrm{~min} .30 \mu \mathrm{g}$ of sample proteins from control cultures were pre-incubated with $10 \mathrm{U}$ calf intestine alkaline phosphatase (Fermentas, Burlington, ON, 
Canada, EF0373), for $30 \mathrm{~min}$ at $37^{\circ} \mathrm{C}$ before electrophoresis, to present a contrast between nonphosphorylated $\mathrm{C} \times 43$ and nonphosphorylated PKCa. Then samples were loaded onto Novex pre-cast gels (Invitrogen). Proteins in the gels were transferred onto Invitrolon PVDF membranes (Invitrogen). WesternBreeze kit (Invitrogen) was applied following immunodetection of Western blots. Meanwhile, we applied two kinds of protein standard, SeeBlue Plus2 and MagicMark XP (Invitrogen) to assertain molecular weight.

Rabbit anti-total Cx43 (Invitrogen-Zymed, South San Francisco, CA, USA; 71-0700) and rabbit anti-N-cadherin (Abcam, ab18203) antibodies were used to investigate the effects of aconitine on $\mathrm{C} \times 43$ phosphorylation status, which were diluted to concentrations of 0.2 and $0.1 \mu \mathrm{g} / \mathrm{ml}$, respectively. And mouse anti-PKCa (Santa Cruz, Santa Cruz, CA, USA; sc-8393), mouse anti-N-cadherin (Zymed, 33-3900), rabbit anti-Ser657 phosphorylated PKCa (Santa Cruz, sc-12356) and rabbit anti-N-cadherin (Abcam, ab18203) antibodies were used to investigate the effects of aconitine on PKCa phosphorylation status. All the primary antibodies were diluted to concentrations of $0.1,0.1,0.2$ and $0.1 \mu \mathrm{g} / \mathrm{ml}$, respectively. X-Omat LS films (Kodak, USA) were used to visualize chemiluminescences of the bands in membranes and band intensities of Cx43 isoforms and Kodak Digital Science 1D image analysis software (Kodak, version 2.0) were used to quantify the densitometry of two kinds of PKCa. Mean values were derived from six independent experiments.

\section{Quantitative immunofluorescent microscopy analysis}

$3.7 \%$ paraformaldehyde-PBS was added to cardiomyocytes grown on bottoms of pre-coated culture wells and kept for $15 \mathrm{~min}$ and then permeabilized with $0.2 \%$ Triton X-100 for $8 \mathrm{~min}$. After Image-iT FX signal enhancer (Invitrogen-Molecular Probes, Eugene, OR, USA) blocking for $30 \mathrm{~min}$, cells were sequentially incubated with rabbit anti-Ser657 PKCa (Abcam, ab30559; diluted 1:200) and rabbit anti-Ser657 phosphorylated PKCa (Zymed, 13-8300; diluted 1:500) for $1 \mathrm{~h}$. Fluorescein-conjugated anti-rabbit $\operatorname{lgG}$ and rhodamineconjugated anti-mouse IgG (Molecular Probes), were used as detection systems (diluted $1: 100$ ) for $30 \mathrm{~min}$. Nuclei were counterstained in Hoechst 33258 solution (Molecular Probes) for 5 min. After washing with TBST and ultrapure water, cells were covered with anti-fade medium. Cultures in the same batch were immunostained simultaneously to eliminate inter-preparative variability; using identical dilutions of primary and secondary antibodies, all of the cultures were detected under the same set of parameters.

The immunoreactive signals of total PKCa and P-PKCa (Ser-657) in cultured cardiomyocytes were examined using an inverted laser scanning confocal microscope (Olympus, Tokyo, Japan; FV500-IX71) at a magnification of $400 x$, with a long working distance objective (Olympus, LUCPLFN 40xPH, 0.60 N.A.). Images were recorded using multi-channel scanning. We took four cultures per experimental group, and analyzed five randomly selected test areas from each culture in order to quantify the PKCa signals. Each test area (about $150 \times 150 \mu^{2}$ ) was digitized into $1024 \times 1024$ pixel array. We defined PKCa positive signal areas as the number of pixels with $\mathrm{PKCa} \mathrm{FI}$ (fluorescent intensity) exceeding the upper threshold of 100 on the $0-255$ gray levels. Pixels with PKCa FI below the lower threshold of 30 were regarded as areas not covered by cells. Most of the cells (>95\%) were proved to be cardiomyocytes in the cultures, and the level of background fluorescence was low. The proportion of PKCa signal positive areas were set as the number of pixels with the $\mathrm{Fl}>100$ divided by the numbers of pixels with the $\mathrm{Fl}>30$ in each test area. The Image-Pro Plus software (Media Cybernetics, Silver Spring, MD, USA; version 5.1) was used for analysis of $\mathrm{PKCa} \mathrm{FI}$.

\section{Statistical analysis}

Experimental data were expressed as $x \pm s$ and SPSS 13.0 was used. Differences of Cx43 isoform or PKCa levels between groups in Western blot studies and proportional differences of Ser368 phosphorylated or nonphosphorylated $\mathrm{Cx} 43$ signal positive areas in immunofluorescence detection were analyzed with univariate two-way analysis of variance (ANOVA); and multiple comparisons were made using the Games-Howell post hoc test. A value of $P<$ 0.05 was considered statistically significant.

\section{RESULTS}

\section{Effects of aconitine on Cx43 phosphorylation status}

As shown in the blot (Figure $1 \mathrm{~A}$ ), a major band at $43 \mathrm{kDa}$, which represented P-Cx43, could be detected in control cultures. When treated with alkaline phosphatase, the band intensity of $43 \mathrm{kDa}$ disappeared. Treatment of the cultures with aconitine resulted in the obvious weakness of the band at $43 \mathrm{kDa}$. While in the representative blot (Figure 1B), the band at $39 \mathrm{kDa}$ comprised the nonphosphorylated isoform of NP-Cx43. Obviously, aconitine induced dephosphorylation of $\mathrm{Cx} 43$ in cultured cardiomyocytes. Comparing such representative blots, the band of $39 \mathrm{kDa}$ showed in the complementary parallels of the band of $43 \mathrm{kDa}$.

As seen from the results of densitometric analysis (Figures $1 \mathrm{C}$ and $\mathrm{D}$ ), incubation of the cultures with $1 \mu \mathrm{M}$ aconitine for $1 \mathrm{~h}$ led to a selective accumulation the level of $\mathrm{P}-\mathrm{Cx} 43$, which had been significantly decreased to $0.37 \pm 0.04(n=6)$, concomitant with a significant increase in the percentage of NP-Cx43 to $4.29 \pm 0.27(n=6)$, compared to the controls ( $n=6$, in Table 1). However, treatment of the cultures with Gö6976 or AAP, whether or not they were followed by aconitine incubation, caused significant increase in phosphorylated $\mathrm{C} \times 43$ levels; mean while the percentage of $\mathrm{P}-\mathrm{C} \times 43$ and NP-Cx43 had not changed significantly compared to the controls. It is apparent from the Cx43 isoform band patterns and the densitometric analysis that pretreatment of the cultures with AAP or Gö6976 could antagonize the dephosphorylation effect of aconitine on cardiomyocytes.

\section{Effects of aconitine on PKCa isoforms phosphorylation status}

As shown in the representative blot (Figures 2A and B), only a major band at $80 \mathrm{kDa}$ could be detected in both the control and the cultures. When treated with aconitine or alkaline phosphatase, the band intensity of $80 \mathrm{kDa}$ strengthened, but it did not showed significant difference. Treatment of the cultures with Gö6976 or AAP resulted in the insignificant intensified appearance of the band at $80 \mathrm{Da}$. Meanwhile, the band at $80 \mathrm{Da}$, comprised the 

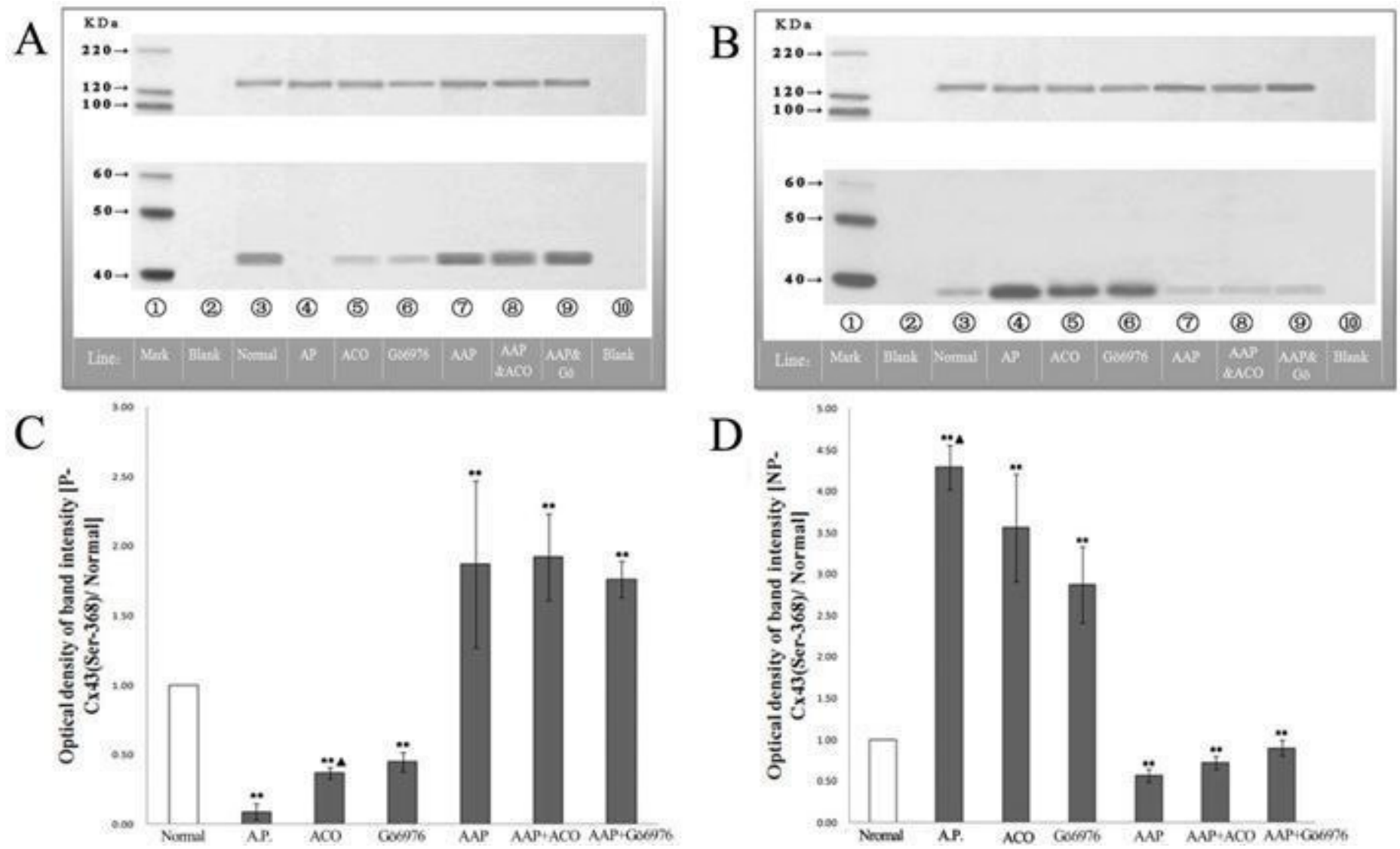

Figure 1. Representative Western blot and quantitative densitometric analysis of $\mathrm{Cx} 43$ protein isoforms in control and drug-treated cultures from six independent experiments. Loading of $\mathrm{P}-\mathrm{C} \times 43$ and NP-Cx43 are presented, respectively in panels $\mathrm{A}$ and $\mathrm{B}$. Cx43 isoform levels were represented by band intensities. Levels of P-Cx43 and NP-Cx43 are presented, respectively in panels C and D. ${ }^{* *}$ Indicates significant differences of $\mathrm{P}<0.01$ compared to control; ${ }^{\wedge}$ Indicates significant differences of $\mathrm{P}<0.01$ compared to aconitine.

Table 1. Quantitative Western blots densitometric analysis of band intensity of P-Cx43 (Ser-368) and NP-Cx43 (Ser-368) protein in culture groups.

\begin{tabular}{|c|c|c|}
\hline Group & $\begin{array}{l}\text { Optical density of band intensity } \\
\text { (P-Cx43(Ser-368)/ Normal) }\end{array}$ & $\begin{array}{l}\text { Optical density of band intensity } \\
\text { [NP-Cx43(Ser-368)/ Normal] }\end{array}$ \\
\hline Normal & 1.00 & 1.00 \\
\hline A.P. & $0.09 \pm 0.06^{* *}$ & $4.29 \pm 0.27^{\star \star \Delta}$ \\
\hline $\mathrm{ACO}$ & $0.37 \pm 0.04^{\star \star \Delta} \Delta$ & $3.56 \pm 0.65^{\star \star}$ \\
\hline Gö6976 & $0.45 \pm 0.07^{* *}$ & $2.87 \pm 0.46^{\star \star}$ \\
\hline AAP & $1.87 \pm 0.60^{* *}$ & $0.57 \pm 0.08^{* *}$ \\
\hline $\mathrm{AAP}+\mathrm{ACO}$ & $1.92 \pm 0.31^{* *}$ & $0.72 \pm 0.08^{\star *}$ \\
\hline$A A P+G o ̈ 6976$ & $1.76 \pm 0.13^{* *}$ & $0.90 \pm 0.09^{* *}$ \\
\hline
\end{tabular}

Values of the relative amount of $\mathrm{Cx} 43$ were defined as a quantitative densitometric analysis of $\mathrm{Cx} 43$ isoforms in normal and drug-treated groups, and the drug-treated rats were taken from six independent experiments. Values are expressed as $\bar{x} \pm s(n=6)$, ${ }^{* *} P<0.01$ vs. control. ${ }^{\mathbf{P}} \mathrm{P}<0.01$ vs. aconitine.

phosphorylated isoform of PKCa (Ser-657), were detected. Obviously, aconitine and Gö6976 weakened the appearance of the band at $80 \mathrm{Da}$, which indicated aconitine and Gö6976 lead to dephosphorylation of PKCa in cultured cardiomyocytes; however, the disappearance could be suppressed by AAP. 

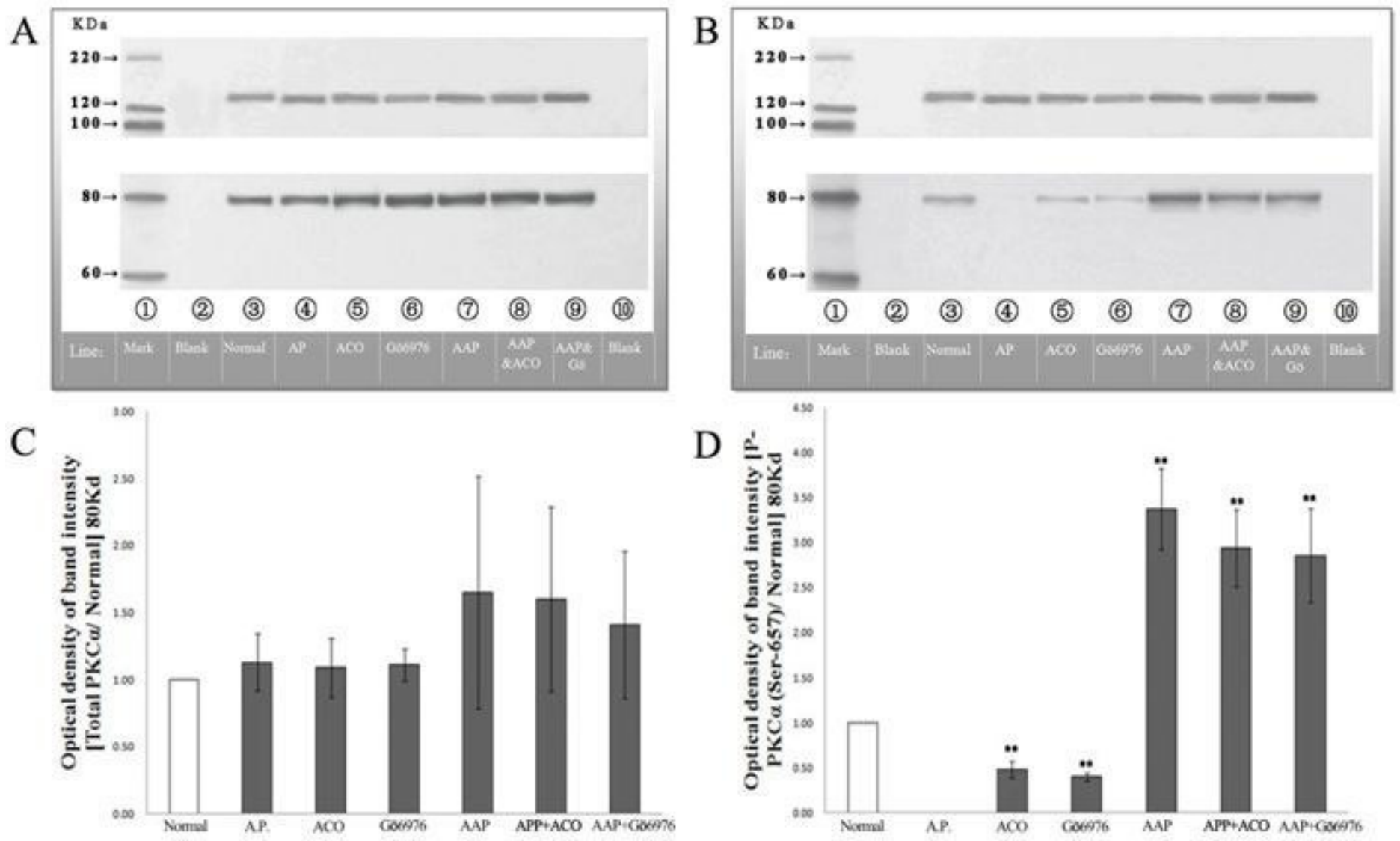

Figure 2. Representative Western blot and quantitative densitometric analysis of PKCa protein isoforms in control and drug-treated cultures from six independent experiments. Loading of total PKCa and P-PKCa are presented, respectively in panels A and B. Cx43 isoform levels were represented by band intensities. Levels of total PKC $\alpha$ and P-PKCa are presented, respectively in panels $C$ and D. ** Indicates significant differences of $\mathrm{P}<0.01$ compared to control.

Table 2. Quantitative Western blots densitometric analysis of band intensity of PKCa and P- PKCa (Ser-657) protein in culture groups.

\begin{tabular}{lcc}
\hline Group & $\begin{array}{c}\text { Optical density of band intensity (Total } \\
\text { PKC } \boldsymbol{\alpha} / \text { Normal) }\end{array}$ & $\begin{array}{c}\text { Optical density of band intensity [P- PKC } \boldsymbol{\alpha} \\
\text { (Ser-657)/ Normal] }\end{array}$ \\
\hline Normal & 1.00 & 1 \\
A.P. & $1.13 \pm 0.21$ & 0 \\
ACO & $1.09 \pm 0.22$ & $0.48 \pm 0.09^{\star *}$ \\
Gö6976 & $1.11 \pm 0.12$ & $0.40 \pm 0.04^{* \star}$ \\
AAP & $1.65 \pm 0.87$ & $3.38 \pm 0.45^{* *}$ \\
AAP+ACO & $1.60 \pm 0.69$ & $2.94 \pm 0.43^{* *}$ \\
AAP+ Gö9676 & $1.41 \pm 0.55$ & $2.86 \pm 0.52^{* *}$ \\
\hline
\end{tabular}

Values of the relative amount of PKCa were defined as a quantitative densitometric analysis of PKCa isoforms in normal and drug-treated groups, and were taken from six independent experiments. Values are expressed as $\bar{x} \pm s(\mathrm{n}=6) .{ }^{* *} \mathrm{P}<0.01$ vs. control.

As seen from the results of densitometric analysis (Figures 2C and D), incubation of the cultures with aconitine led to a nonspecific accumulation the level of total PKCa, which had been insignificantly increased to $1.09 \pm 0.22(n=6)$, concomitant with a significant decrease in the percentage of P-PKCa (Ser-657) to $0.48 \pm 0.09(n=6)$, compared to the controls ( $n=6$, in Table 2). However, treatment of the cultures with Gö6976 or AAP, whether or not they were followed by aconitine incubation, caused significant increase in P-PKCa (Ser-657) levels. It is 

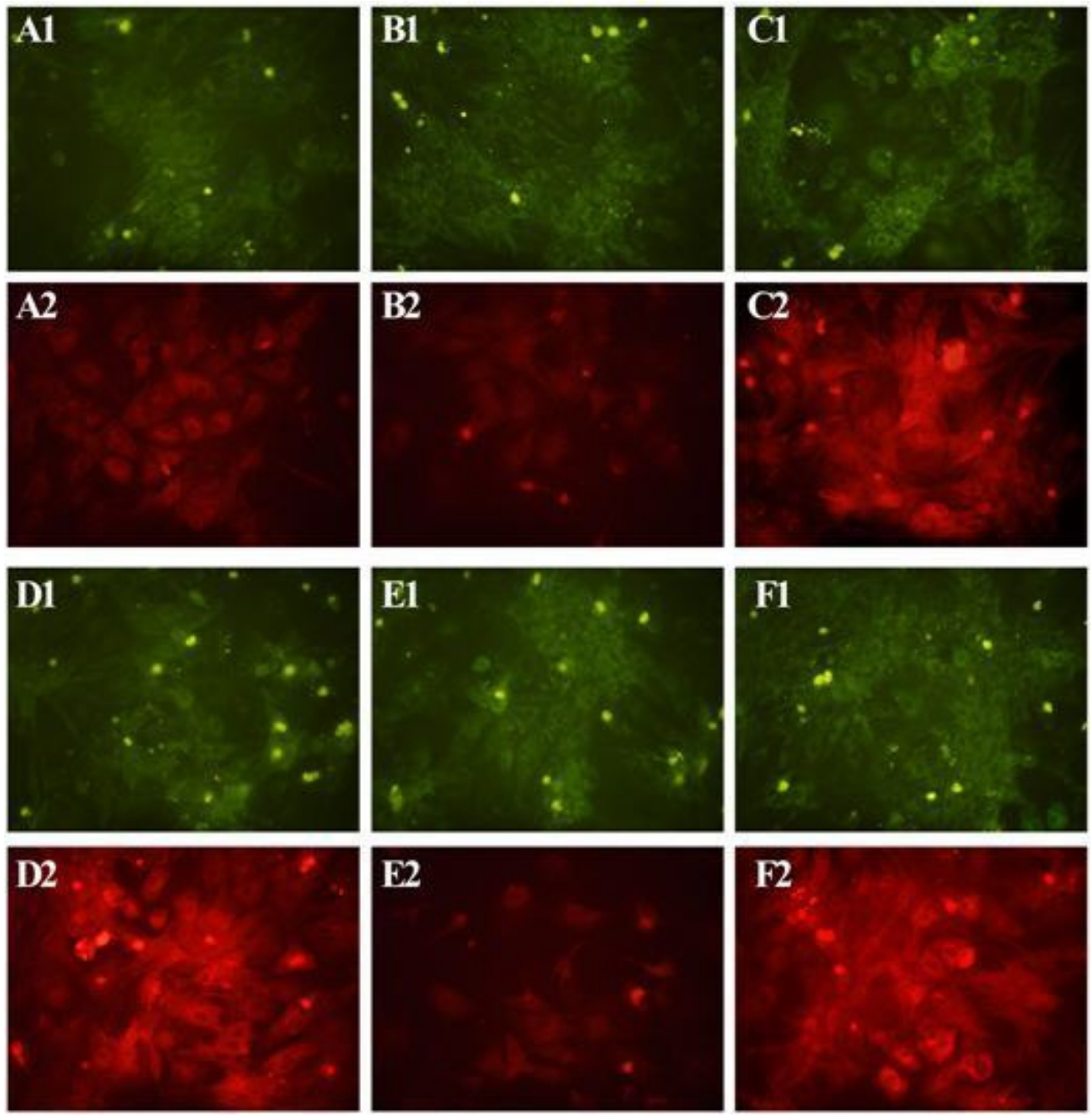

Figure 3. Representative immunofluorescent confocal images of total KCa (Ser657, showed in green fluorescence) and P-PKCa (showed in red fluorescence), came from the experiments. Individual panels are: (A1 and A2) control; (B1 and B2) culture incubated with $1 \mu \mathrm{M}$ aconitine for $1 \mathrm{~h}$; (C1 and C2) culture treated with $0.1 \mu \mathrm{g} / \mathrm{ml}$ AAP for $4 \mathrm{~h}$; (D1 and D2) culture pretreated with AAP for $4 \mathrm{~h}$, followed by $1 \mathrm{~h}$ of aconitine incubation; (E1and E2) culture treated with $0.1 \mu \mathrm{g} / \mathrm{ml}$ Gö6976 for $4 \mathrm{~h}$; (F1 and F2) culture co-incubated with $0.1 \mu \mathrm{g} / \mathrm{ml}$ AAP and $0.1 \mu \mathrm{g} / \mathrm{ml}$ Gö6976 for $4 \mathrm{~h}$. Original magnification is 200x.

apparent from the PKCa isoform band patterns and the densitometric analysis that pretreatment of the cultures with AAP could antagonize the dephosphorylation effect of aconitine on cardiomyocytes.

It is apparent that the amount of PKC $\alpha$ did not change after treatment of the cardiomyocytes with alkaline phosphatase, aconitine or AAP, compared to the controls. However, after aconitine treatment, the amount of P-PKCa (Ser657) decreased significantly, and the band of P-PKCa (Ser657) could hardly be detected in alkaline phosphatase treated cultures. Still, the aconitine-induced dephosphorylation effect on P-PKCa (Ser657) could be antagonized by pretreatment with AAP.

\section{Immunofluorescence of PKCa isoforms phosphorylation status}

The aforementioned studies indicated that aconitine induced P-PKCa (Ser-657) dephosphorylation in cultured cardiomyocytes, and that it could be antagonized by AAP; how these changes occur at PKCa isoforms was revealed through immunofluorescent staining. Under control conditions, P-PKCa (Ser-657) positive plaques were red, sparse Rhodamine, appearing in the cytoplasm of cardiomyocytes (Figure 3, A2). In contrast, total PKCa positive signal were green, spread Fluorescein, also in the cytoplasm (Figure 3 and A1). Incubation of the cultures 
Table 3. Quantitative immunofluorescent analysis of PKCa and P- PKCa (Ser-657) protein in culture groups.

\begin{tabular}{lcc}
\hline Group & PKC $\alpha$ (\%cell area) & P-PKC $\alpha$ (Ser-657)(\%cell area) \\
\hline Control & $72.31 \pm 0.16$ & $0.68 \pm 0.05$ \\
ACO & $71.53 \pm 0.27$ & $0.61 \pm 0.11$ \\
AAP & $73.65 \pm 0.19$ & $3.81 \pm 0.53^{\star *}$ \\
AAP+ACO & $74.19 \pm 0.38$ & $3.17 \pm 0.67^{\star *}$ \\
Gö6976 & $71.76 \pm 0.29$ & $0.6 \pm 0.09$ \\
AAP+ Gö6976 & $73.85 \pm 0.31$ & $3.01 \pm 0.43^{\star *}$ \\
\hline
\end{tabular}

Values of the relative amount of PKCa were defined as a percentage of the pixels in cell area occupied by PKCa optical density, and were taken from six independent experiments. Values are expressed as $\bar{x} \pm s(\mathrm{n}=20)$. ${ }^{* *} \mathrm{P}<0.01$ vs. control.

with aconitine $(1 \mu \mathrm{M}$, for $1 \mathrm{~h})$ led to a dramatic loss of P-PKCa (Ser-657) (Figure 3, B2), whereas the total PKCa particles were found insignificantly different (Figure 3, A2). When the cultures were treated with AAP $(0.1 \mu \mathrm{g} / \mathrm{ml}$, for 4 h), prominent P-PKCa (Ser-657) positive patches were observed significantly increased, no matter whether followed by aconitine or Gö6976 incubation or not (Figures 3, C2, D2 and F2); and the total PKCa signals in cultures with parallel treatment were faint (Figures 3, C1, D1 and F1). Incubation of the cultures with Gö6976 (0.1 $\mu \mathrm{g} / \mathrm{ml}$, for $4 \mathrm{~h}$ ) led to a dramatic loss of P-PKCa (Ser-657) (Figure 3, E2), the same as the incubation of aconitine; whereas the total PKCa particles were found insignificantly different with control and aconitine treated, whether co-incubation with AAP (Fig.ure 3, E1). Under AAP and Gö6976 co-incubation, a dramatic increase was observed, the same as the AAP treat. Quantitative immunofluorescent data are presented in Table 3.

In accord, with the results of Western blot and immunofluorescence, these findings suggested that PKCa phosphorylation/dephosphorylation changes are associated with cardiac gap junctions.

\section{DISCUSSION}

Previous researches indicated that aconitine can bind to the open state of voltage-gated sodium channels with high affinity, by facilitating excitation of the membrane, increasing $\mathrm{Na}^{+}$influx and prolonging depolarization (Amran et al., 2004; Kumar et al., 1996). Such properties brought about various types of ventricular tachyarrhythmia or early and delayed after-depolarization in either the animal models or single ventricular myocytes (Guerrero et al., 1997; Zeevi-Levin et al., 2005). However, it is not known whether aconitine has any effects on the connexin43 phosphorylation status and PKCa expression in cardiomyocytes. The results in the study may prove constructive in solving this issue.

\section{Effects of aconitine on the phosphorylation status of Cx43 in cardiomyocytes}

Cx43 is a phosphoprotein, which alter the phosphorylation status at specific sites in the carboxyl terminal. Such sites involved in not only regulating the permeability and conductance of gap junction channels, but also the trafficking, assembly/disassembly, degradation of $\mathrm{C} \times 43$ and other intracellular processes (Bornancin et al., 1997; TenBroek et al., 2001; Joell et al., 2005). In previous experimental models, cardiac Cx43 got dephosphorylated under condition of ischemia, hypoxia and glucose/ATP depletion (Turner et al., 2004; Matsushita et al., 2006), through the regulation of protein kinase deactivation or protein phosphatase activation.

Now, most of the 14 serine and two of the tyrosine residues from different protein kinases are known to be phosphorylated and dephosphorylated at such status (Lampe and Lau, 2004; Thomas et al., 1998). In our abovementioned study, Cx43 was testified underwent significant dephosphorylation at Ser368 in cultured cardiomyocytes after aconitine incubation. The result verifies that aconitine does indeed alter $\mathrm{C} \times 43$ phosphorylation status by some certain mechanisms. A most important phosphorylation site in the carboxyl terminal of Cx43 is a protein kinase C (PKC) site, Ser368 (Dempsey et al., 2000). According to the studies, under condition of ischemia or hypoxia, dephosphorylation of Ser368 in removed rat hearts could contribute to electrical uncoupling and a decrease in rate-pressure adjustment.

AAP, a naturally occurring antiarrhythmic peptide, could improve rhythmicity of cultured cardiomyocytes and several in vivo models of arrhythmia, including aconitine induced arrhythmias (Weng et al., 2002; Müller et al., 1997; Dhein et al., 2001). In our experiments, pretreatment of the cultures with AAP do prevent aconitine-induced Cx43 dephosphorylation effectively. One of the effects of AAP is to activate PKC, especially a conventional isoform PKCa; and the effect lead to 
phosphorylation of the cardiac gap junction protein $\mathrm{Cx} 43$, which provides a promotion of cellular coupling (Müller et al., 1997). Therefore, once the lipid-soluble aconitine get through the cytomembrane, the drug probably acts on PKCa, with subsequent $\mathrm{Cx} 43$ dephosphorylation.

There were researches indicated that the major changes in the degradation of connexins are not linked to their phosphorylation status. Though the phosphorylation is not a generic prerequisite for connexin degradation, phosphorylation of $\mathrm{Cx} 43$ may trigger internalization and degradation in mitotic cells (Thomas et al., 1998; Parekh et al., 2000; Ek-Vitorin et al., 2006). In our study, treatment of the cultures with AAP resulted in an increase of phosphorylated $\mathrm{C} \times 43$ and the prevention of aconitine or Gö6976-induced Cx43 dephosphorylation (Figure 1). Since Gö6976 is representative inhibitors for PKCa, the mechanisms underling the observations may consist in the degradation of phosphorylated $\mathrm{C} \times 43$ being associated with PKCa.

\section{Effects of aconitine on the phosphorylation status of PKCa in cardiomyocytes}

PKC, a family of serine/threonine kinases, is comprised at least 12 different isoforms belong to subgroups based on structure and cofactor requirements. The main function of PKC isoforms is to participate in intracellular signal transduction and regulate a wide range of activities (Dhein et al., 2001). Three conserved residues in PKCa kinase domain could regulate its kinase activity by phosphorylation, which are the hydrophobic C-terminal site Ser657, the activation loop site Thr497 and the autophosphorylation site Thr638. Without phosphorylation at Thr638, Ser657 and Thr497, PKCa has little or no activity (Bornancin et al., 1997; TenBroek et al., 2001).

According to Western blotting, the results indicate that the amount of total PKCa did not change after aconitine treatment, while the amount of P-PKCa (Ser657) decreased significantly (Figure 2). The previous research has demonstrated that the phosphorylation of Ser657 is rate-limiting for the PKCa phosphorylation and it is required for the efficiency of the first and second phase of PKCa. Besides, the phosphorylation of PKCa contributes to be "bottleneck" in a closed phosphatase-resistant conformation completely (Dhein, 2004).

In the present study, we found that the AAP pretreatment could be effectively prevent aconitine-induced dephosphorylation effects on Cx43 (Ser368) and PKCa (Ser657). In addition, a synthetic derivation, AAP10 is reported to be able to activate PKCa, which induce a further phosphorylation of Cx43 (Müller et al., 1997). We therefore, infer that aconitine-induced dephosphorylation of PKCa (Ser657) is correlative with the occurrence of Cx43 (Ser368) dephosphorylation, or somehow a premise for subsequent outcome.
The immunofluorescence demonstrated the same effects of aconitine on the phosphorylation status of $\mathrm{Cx} 43$ at Ser368 and PKCa at Ser657 as in the West blotting. The PKCa could be activated by AAP, leading to Cx43 phosphorylation; furthermore, it proves that the activity of PKCa is sensitive to the frequency of the $\mathrm{Ca}^{2+}$ oscillation. However, it remains unclear whether other serine or threonine phosphorylation sites in the carboxyl terminal of Cx43 or PKCa could be affected by aconitine-treated neonatal ventricular myocytes. Whether the reality is consistent with the results found in the cultured cardiomyocytes also remains a question. Hopefully, future studies will clarify these questions.

\section{ACKNOWLEDGEMENT}

The first two authors (Yan Liu and Man Liang) contributed equally to this work.

\section{REFERENCES}

Amran MS, Hashimoto K, Homma N (2004). Effects of sodium-calcium exchange inhibitors, KB-R7943 and SEA0400, on aconitine-induced arrhythmias in guinea pigs in vivo, in vitro, and in computer simulation studies. J. Pharmacol. Exp. Ther., 310: 83-89.

Axelsen LN, Stahlhut M, Mohammed S, Larsen BD, Nielsen MS, Holstein-Rathlou NH, Andersen S, Jensen ON, Hennan JK, Kjølbye AL (2006). Identification of ischemia-regulated phosphorylation sites in connexin43: a possible target for the antiarrhythmic peptide analogue rotigaptide (ZP123). J. Mol. Cell. Cardiol., 40: 790-798.

Baselt RC (2004). Disposition of Toxic Drugs and Chemicals in Man, 7th ed. Biomedical publications, Foster City, pp. 357-363.

Beardslee MA, Lerner DL, Tadros PN, Laing JG, Beyer EC, Yamada KA, Kléber AG, Schuessler RB, Saffitz JE (2000). Dephosphorylation and intracellular redistribution of ventricular connexin43 during electrical uncoupling induced by ischemia. Circ. Res., 87: 656-662.

Bornancin F, Parker PJ (1997). Phosphorylation of protein kinase C- $\alpha$ on serine 657 controls the accumulation of active enzyme and contributes to its phosphatase-resistant state. J. Biol. Chem., 272: 3544-3549.

Chan TY (2009). Aconite poisoning presenting as hypotension and bradycardia. Hum. Exp. Toxicol., 28: 795-797.

Dempsey EC, Newton AC, Mochly-Rosen D, Fields AP, Reyland ME, Insel PA, Messing RO (2000). Protein kinase C isozymes and the regulation of diverse cell responses. Am. J. Physiol-Lung C., 279: 429-438.

Dhein S (2004). Pharmacology of gap junctions in the cardiovascular system. Cardiovasc. Res., 62: 287-298.

Dhein S, Polontchouk L, Salameh JA (2002). Pharmacological modulation and differential regulation of the cardiac gap junction proteins connexin43 and connexin40. Bio. Cell, 94: 409-422.

Dhein S, Weng S, Grover R, Tudyka T, Gottwald M, Schaefer T, Polontchouk $L$ (2001). Protein kinase $C$ alpha mediates the effect of antiarrhythmic peptide on gap junction conductance. Cell. Commun. Adhes., 8: 257-264.

Dhein S, Weng S, Polontehouk L, Grover R (2001). Pharmacological modification of gap junctional coupling by antiarrhythmic peptides Role of PKC. Naunyn Schmiedeberg' s Arch Pharmacol., 363 Suppl: R99.

Ek-Vitorin JF, King TJ, Heyman NS, Lampe PD, Burt JM (2006). Selectivity of connexin43 channels is regulated through protein kinase C-dependent phosphorylation. Circ. Res., 98: 1498-1505.

Elliott SP (2002). A case of fatal poisoning with the aconite plant: 
quantitative analysis in biological fluid. Sci. Justice, 42: 111-115.

Giepmans BN (2004). Gap junctions and connexin-interacting proteins. Cardiovasc. Res., 62: 233-245.

Guerrero PA, Schuessler RB, Davis LM, Beyer EC, Johnson CM, Yamada KA, Saffitz JE (1997). Slow ventricular conduction in mice heterozygous for a connexin 43 null mutation. J. Clin. Invest., 99: 1991-1998.

Joell LS, Paul DL (2005). Connexin phosphorylation as a regulatory event linked to gap junction channel assembly. Biochimica. et Biophysica. Acta., 1711: 154-163.

Kumar NM, Gilula NB (1996). The gap junction communication channel. Cell, 84: 381-388.

Lampe PD, Lau AF (2004). The effects of connexin phosphorylation on gap junctional communication. Int. J. Biochem. Cell Biol., 36: 1171-1186.

Li M, Wang J, Xie HH, Shen FM, Su DF (2007). The susceptibility of ventricular arrhythmia to aconitine in conscious Lyon hypertensive rats. Acta. Pharmacol. Sin., 28: 211-215.

Lin H, Ogawa K, Imanaga I, Tribulova N (2006). Alterations of connexin 43 in the diabetic rat heart. Adv. Cardiol., 42: 243-254.

Liu Y, Zhang S, Liang M, Liu Q, Liu L (2008). Effects of aconitine on $\left[\mathrm{Ca}^{2+}\right]$ oscillation in cultured myocytes of neonatal rats. J. Huazhong Univ. Sci. Tech., 28: 499-503.

Matsushita S, Kurihara H, Watanabe M, Okada T, Sakai T, Amano A (2006). Alterations of phosphorylation state of connexin43 during hypoxia and reoxygenation are associated with cardiac function. $\mathrm{J}$. Histochem. Cytochem., 54: 343-353.

Moric E, Herbert E, Trusz-Gluza M, Filipecki A, Mazurek U, Wilczok T (2003). The implications of genetic mutations in the sodium channel gene (SCN5A). Europace, 5: 325-334.

Müller A, Gottwald M, Tudyka T, Linke W, Klaus W, Dhein S (1997). Increase in gap junction conductance by an antiarrhythmic peptide. Eur. J. Pharmacol., 327: 65-72.

Müller A, Schaefer T, Linke W, Tudyka T, Gottwald M, Klaus W, Dhein S (1997). Actions of the antiarrhythmic peptide AAP10 on intercellular coupling. Naunyn Schmiedebergs Arch Pharmacol., 356: 76-82.

Parekh DB, Ziegler W, Parker PJ (2000). Muliple pathways control protein kinase C phosphorylation. EMBO J., 19: 496-503.

Pullela R, Young L, Gallagher B, Avis SP, Randell EW (2008). A Case of fatal aconitine poisoning by monkshood ingestion. J. Forensic Sci., 53 491-494.

Sáez JC, Retamal MA, Basilio D, Bukauskas FF, Bennett MV (2005). Connexin-based gap junction hemichannels: gating mechanisms. Biochim. Biophys. Acta., 1711: 215-224.

Salameh A (2006). Life cycle of connexins: regulation of connexin sythesis and degradation. Adv. Cardiol., 42: 57-70.
Schulz R, Heusch G (2004). Connexin43 and ischemic preconditioning. Cardiovasc. Res., 62: 335-344.

Sohl G, Willecke K (2004). Gap junctions and the connexin protein family. Cardiovasc. Res., 62: 228-232.

Stahlhut M, Petersen JS, Hennan JK, Ramirez MT (2006). The antiarrhythmic peptide rotigaptide (ZP132) increases connexin 43 protein expression in neonatal rat ventricular cardiomyocytes. Cell. Commun. Adhes., 13: 21-27.

TenBroek EM, Lampe PD, Solan JL, Reynhout JK, Johnson RG (2001). Ser364 of connexin43 and the upregulation of gap junction assembly by cAMP. J. Cell. Biol., 155: 1307-1318.

Thomas SA, Schuessler RB, Berul Cl, Beardslee MA, Beyer EC, Mendelsohn ME, Saffitz JE (1998). Disparate effects of deficient expression of connexin43 on atrial and ventricular conduction: evidence for chamber-specific molecular determinations of conduction. Circulation, 97: 686-691.

Turner MS, Haywood GA, Andreka P, You L, Martin PE, Evans WH, Webster KA, Bishopric NH (2004). Reversible connexin43 dephosphorylation during hypoxia and reoxygenation is linked to cellular ATP levels. Circ. Res., 95: 726-733.

Wang SY, Wang GK (2003). Voltage-gated sodium channels as primary targets of diverse lipid-soluble neurotoxins. Cell Signal, 15: 151-159.

Weng S, Lauven M, Schaefer T, Polontchouk L, Grover R, Dhein S (2002). Pharmacological modulation of gap junction coupling by an antiarrhythmic peptide via protein kinase $\mathrm{C}$ activation. FASEB J., 16: 1114-1116.

Xia Y, Gong KZ, Xu M, Zhang YY, Guo JH, Song Y, Zhang P (2009). Regulation of gap-junction protein connexin 43 by beta-adrenergic receptor stimulation in rat cardiomyocytes. Acta. Pharmacol. Sin., 30: 928-34.

Zeevi-Levin N, Barac YD, Reisner Y, Reiter I, Yaniv G, Meiry G, Abassi Z, Kostin S, Schaper J, Rosen MR, Resnick N, Binah O (2005). Gap junctional remodeling by hypoxia in cultured neonatal rat ventricular myocytes. Cardiovasc. Res., 66: 64-73. 